\title{
Characterization of the biofilm forming ability of Staphylococcus pseudintermedius from dogs
}

\author{
Ameet Singh ${ }^{1 *}$, Meagan Walker ${ }^{1}$, Joyce Rousseau ${ }^{2}$ and Jeffrey Scott Weese ${ }^{2}$
}

\begin{abstract}
Background: Staphylococcus pseudintermedius is an opportunistic pathogen of dogs and has emerged as a leading cause of skin, wound and surgical site infections worldwide. Methicillin resistance is common and clinical infections as a result of methicillin-resistant S. pseudintermedius (MRSP) pose a clinical challenge. In other staphylococci, biofilm formation has been shown to be a virulence factor for infection, however, it has received little attention in $S$. pseudintermedius. The objectives of this study were to evaluate the biofilm forming ability of clinical isolates of $S$. pseudintermedius obtained from dogs using phenotypic and genotypic techniques.

Results: $96 \%$ (136/140) of S. pseudintermedius isolates were classified as strong or moderate biofilm producers, with the majority of isolates being able to produce biofilm. There was no difference in biofilm formation between MRSP and MSSP ( $p=0.8$ ), amongst isolates from clinical infections compared with isolates obtained from colonized dogs ( $p=0.08$ ), and between isolates from sequence type (ST) 71 and ST 68 ( $P=0.09$ ). icaA was detected in 77.9\% (109/140) of isolates and icaD was detected in $75.7 \%$ (106/140) of isolates. Scanning electron microscopic evaluation of S. pseudintermedius biofilm production revealed aggregates of cocci and irregularly produced extracellular polymeric matrix.

Conclusion: The majority of S. pseudintermedius isolates evaluated in this study were able to produce biofilm and this may be an important virulence factor in the rapid emergence of this bacterium in veterinary hospitals worldwide. Further study into the mechanisms of biofilm formation by S. pseudintermedius is warranted.
\end{abstract}

Keywords: Biofilm, Staphylococcus pseudintermedius, Microtitre plate assay, icaA, icaD

\section{Background}

Surgical site infections (SSI) are an inherent risk of any surgical procedure and are reported as a complication in $0.8 \%$ to $18.1 \%$ of operations depending on wound classification [1-4]. The consequences of SSI in veterinary medicine include patient morbidity/mortality, prolonged hospitalization, increased treatment cost, frustration and grief of pet owners and caregivers alike. In veterinary surgical patients, SSI are becoming complicated by the emergence of multi-drug resistant bacteria as these infections are challenging to treat due to their resistance to many of the commonly used antimicrobials [5,6]. Of particular concern in dogs and cats has been the rapid emergence of methicillin-resistant Staphylococcus pseudintermedius (MRSP) [6]. This multidrug resistant opportunistic pathogen has spread rapidly and widely in recent years [6-8],

\footnotetext{
*Correspondence: amsingh@uoguelph.ca

'Department of Clinical Studies, Ontario Veterinary College, University of Guelph, Guelph, ON N1G 2W1, Canada

Full list of author information is available at the end of the article
}

and is the most common cause of SSI in some veterinary facilities [9].

Implant-related SSI are a serious complication and frequently result in implant removal since routine antimicrobial administration is rarely effective [10-13]. Implant removal results in additional patient morbidity, increased treatment costs, and, in some cases, may require re-application of a surgical implant [10-13]. It has been well documented that implant-related SSI in humans are complicated by the presence of bacterial biofilms [14,15]. A bacterial biofilm is a complex, sessile community of bacteria embedded within a self-produced matrix of carbohydrates, proteins and DNA (extracellular polymeric substance, EPS) [16-19]. Within a biofilm, bacteria have markedly altered metabolism, enhanced cell-to-cell communication, and are able to evade the host immune response and the effects of antimicrobials through their isolated metabolism along with physical and chemical protection of the biofilm matrix [17-19]. Following placement of an implant, it is rapidly coated 
with a host-derived, protein-based conditioning film, which contains receptors that allow for bacterial attachment initiating the process for biofilm formation [17-19]. The ability of bacteria to form a biofilm has been shown to be a leading cause of persistent SSI and, thus, the presence of a biofilm can greatly impact the ability to treat an SSI [17-19].

Biofilm formation is now recognized as an important virulence factor in several Staphylococcus spp. [20,21]. The ability to form a biofilm is likely variable between bacterial species, and the biofilm forming ability of $S$. pseudintermedius has not been fully characterized. One small report involving 23 MRSP isolates from dogs in Norway revealed that all isolates were biofilm producers with isolates belonging to sequence type (ST) 71 producing significantly more biofilm compared with other STs [22]. Another report revealed that clarithromycin was ineffective in eradicating MRSP biofilm at therapeutic doses [23]. In that study, all 20 MRSP isolates evaluated formed biofilm [23]. As MRSP has now become the leading cause of SSI in veterinary medicine, characterizing its biofilm forming ability will provide further insight into a rapidly emerging clinical dilemma.

Once initial attachment to a biomaterial occurs, a key step in biofilm formation is secretion of EPS [19]. In staphylococci, this extracellular "slime" component, also termed polysaccharide intercellular adhesion (PIA), is encoded, at least in part, by the ica operon $[24,25]$. In S. epidermidis, it has been shown that co-expression of icaA and icaD leads to an increase in the activity of PIA [25]. However, icaindependent biofilm formation has been reported in staphylococci [26] and the role of the ica operon in the biofilm forming ability of $S$. pseudintermedius is unknown.

The objectives of this study were to evaluate the biofilm forming ability of clinical isolates of S. pseudintermedius obtained from dogs using a quantitative microtitre plate assay (MPA), to compare biofilm formation amongst strains, and to determine the presence and impact of biofilm-associated genes (icaA and icaD).

\section{Methods}

\section{Isolates}

One-hundred and twenty one MRSP and 19 methicillinsusceptible S. pseudintermedius (MSSP) obtained from dogs were used. Isolates were obtained from a convenience sample of isolates from clinical infection or colonization in dogs from Canada and the United States. The isolates were collected from 2005 to 2012 from clinical cases and surveillance studies. The isolates were stored at $-80^{\circ} \mathrm{C}$ in Cryostor beads (Innovatek Medical, Delta, BC).

\section{Phenotypic characterization of biofilm formation}

Biofilm formation was evaluated using a quantitative spectrophotometric microtitre plate assay (MPA) as previously described [27]. Briefly, isolates were subcultured onto blood agar and pure $24 \mathrm{hr}$ growth used. Each isolate was suspended in $5.0 \mathrm{ml}$ of tryptic soy broth (TSB) supplemented with $1 \%$ glucose to achieve a turbidity equivalent to a 0.5 McFarland-standard $\left(\sim 10^{8}\right.$ $\mathrm{CFU} / \mathrm{ml}$ ). A $200 \mu \mathrm{l}$ bacterial suspension was inoculated into a 96-well microtitre plate in triplicate and incubated overnight for $24 \mathrm{hr}$ at $35^{\circ} \mathrm{C}$ without shaking to allow biofilm formation. Following incubation, the contents of the wells were discarded and each well was washed three times with sterile phosphate buffered saline (PBS) $(\mathrm{pH}$ 7.2) to remove non-adherent (planktonic) cells while carefully ensuring the integrity of formed biofilms was maintained. The adhered bacteria (biofilm) were heat fixed for $60 \mathrm{~min}$ at $60^{\circ} \mathrm{C}$. Adhered cells were dyed with $150 \mu \mathrm{l}$ of $0.1 \%(\mathrm{w} / \mathrm{v})$ crystal violet for $15 \mathrm{~min}$ at $22^{\circ} \mathrm{C}$ and air dried. Following re-solubilization with $95 \%$ ethanol, optical density (OD) of each well was measured at $570 \mathrm{~nm}$. Median $\mathrm{OD}_{570}$ of the triplicates of the negative control (TSB only) was subtracted from the median $\mathrm{OD}_{570}$ of triplicates of the samples. Based on the report by Stepanovich et al. [27], isolates were classified as strong, moderate, weak or zero biofilm producers based on their $\mathrm{OD}_{570}\left(4 \times\right.$ ODc $<\mathrm{OD}_{570}=$ strong biofilm producer, $2 \times$ ODc $<\mathrm{OD}_{570} \leq 4 \times$ ODc $=$ moderate biofilm producer, ODc $<\mathrm{OD}_{570} \leq 2 \times$ ODc $=$ weak biofilm producer, $\mathrm{OD}_{570} \leq \mathrm{ODc}=$ no biofilm producer (ODcutoff $(\mathrm{ODc})=$ average $\mathrm{OD}_{570}$ of negative control + $(3 \times$ stand ard deviation of negative control)).

\section{DNA extraction}

Isolates were grown overnight on Colombia Blood agar and incubated at $35^{\circ} \mathrm{C}$. A sample of a single colony $(\sim 10 \mu \mathrm{l})$ was re-suspended in $1 \mathrm{ml}$ sterile water. The suspension was centrifuged at 12,000 rpm for 2 minutes. The supernatant was discarded and $200 \mu \mathrm{l}$ of BIO-RAD InstaGene Matrix (Bio-Rad Laboratories, Montreal, Canada) was added to the pellet. The suspension was vortexed for 10 seconds and then incubated at $56^{\circ} \mathrm{C}$ for 30 minutes in a water bath. The suspension was then vortexed for 10 seconds and heated in a block heater for 8 minutes at $100^{\circ} \mathrm{C}$. After heating, the suspension was centrifuged at $12,000 \mathrm{rpm}$ for 2 minutes. The supernatant was then transferred to clean $200 \mu \mathrm{l}$ PCR tubes. The extract was stored at $-20^{\circ} \mathrm{C}$.

\section{Real-time PCR detection of icaA and icaD genes}

Real time polymerase chain reaction (RT-PCR) was performed in a DNA thermal cycler (CFX96 Real-Time system Thermocycler, Bio-Rad Laboratories Ltd, Montreal, Canada) and used to detect ica A and icaD in all isolates $[28,29]$. Gene sequences for icaA and icaD in Staphylococcus pseudintermedius were obtained from the National Centre for Biotechnology Information GenBank database 
(accession numbers: NC_014925.1 and NC_017568.1, respectively). The primer sequences used for RT-PCR for icaA were forward: 5'- TTGCCCACCTTGTGCCCACC-3' and reverse: 5' TGAGGCTGTAGGGCGTTGGGA-3' and for icaD were forward: 5'- AGACGACACACCCTATG GCTATGAA-3' and reverse: 5'- ACGTATTAGCGCACA TTCGGTGTTA-3'. Reactions were carried out separately for icaA and icaD. The PCR reaction volume was $20 \mu \mathrm{l}$ and contained $10 \mu \mathrm{l}$ RT-PCR super mix (Bio-Rad SSoFast EvaGreen supermix, Bio-Rad Laboratories Ltd, Montreal, Canada) $0.5 \mu \mathrm{l} \mathrm{MgCl}_{2}(2.5 \mathrm{mM}), 1 \mu \mathrm{l}$ forward primer, $1 \mu \mathrm{l}$ reverse primer, $5.5 \mu \mathrm{l}$ sterile water, $2 \mu \mathrm{l}$ bacterial DNA. Thermal cycling conditions were: an initial denaturation at $95^{\circ}$ for $3 \mathrm{~min}$, followed by 40 cycles of 10 seconds of denaturation at $95^{\circ}, 10$ seconds of annealing at $60^{\circ} \mathrm{C}, 30 \mathrm{sec}-$ onds of extension at $72^{\circ}$. After the amplification cycles were complete, a melting curve analysis followed by ramping from $65^{\circ} \mathrm{C}$ to $95^{\circ} \mathrm{C}$ for 5 seconds. Samples with a crossing point $(\mathrm{Ct}<38)$ and a single melting peak consistent with the positive control were considered positive. Positive and negative controls were included with all runs. Representative PCR products of both ica $\mathrm{A}$ and $i c a \mathrm{D}$ were sequenced to confirm identity of peaks in RT-PCR reactions.

\section{Biofilm structure evaluation by scanning electron microscopy (SEM)}

Overnight culture of a strong biofilm producing MRSP isolate was inoculated with TSB $+1 \%$ glucose. A $316 \mathrm{~L}$ stainless-steel $20 \mathrm{~mm}$ orthopaedic bone screw (Veterinary Orthopedic Implants, St. Augustine, FL, USA) was added to $5 \mathrm{ml}$ of a 0.5 McFarland standard suspension and incubated for $24 \mathrm{hrs}$ aerobically at $35^{\circ} \mathrm{C}$. Following incubation, the screw was washed with PBS and fixed at $22^{\circ} \mathrm{C}$ with $2.5 \%$ glutaraldehyde until time of SEM imaging. The day prior to image acquisition, the screw was post-fixed with $1 \%$ osmium tetroxide for $30 \mathrm{~min}$ at $22^{\circ} \mathrm{C}$, washed in Sorensen's phosphate buffer twice for $15 \mathrm{~min}$, dehydrated through an ethanol series $(50 \%, 70 \%, 80 \%$, 90\%, 99.5\% for 15 min each), critical point dried and sputter coated with gold. The screw was then imaged using a Hitachi S-570 SEM. Images were subjectively evaluated for adherent cells and extracellular matrix.

\section{MRSP characterization}

MRSP isolates were characterized by sequence analysis of the mec-associated direct repeat unit (dru typing) [30]. The Dru repeats and types were assigned by the Dru-typing.org database (http://www.dru-typing.org/ search.php). A minimum spanning tree was generated using BioNumerics v6.6 (Applied Maths, Austin, Texas, USA) and the TRST plugin. Distance intervals were created using a bin distance of $1.0 \%$. Dru types separated by an MST distance of $<2$ ( $>98.5 \%$ similarity) were considered closely related and assigned to the same cluster
[31]. The root node was assigned to the sequence type with the greatest number of isolates.

\section{Statistical analysis}

Using commercially available software (JMP statistical discovery, Cary, NC, USA), descriptive statistics were performed and Student's $t$-test used for continuous outcome data while Fisher's exact and chi-square tests were used for categorical comparisons. Logistic regression analysis was also performed. A $p$ value of $<0.05$ was considered significant.

\section{Results}

Ninety-six percent (136/140) of S. pseudintermedius isolates were classified as strong or moderate biofilm producers, with the majority of isolates being able to produce biofilm to some degree (Figure 1). No difference in biofilm formation between MRSP and MSSP $(\mathrm{p}=0.8)$ was noted (Table 1). Ninety-three percent (132/140) of isolates belonged to two dru clusters, rooted by $\mathrm{dt} 9 \mathrm{a}(\mathrm{n}=58,48 \%)$ and dt11a $(\mathrm{n}=55,45 \%)$ [32]. These correspond to the two major MRSP clonal complexes, sequence type (ST) 71 and ST 68, respectively. No significant difference in biofilm formation between these two clonal complexes $(\mathrm{P}=0.09)$ was observed (Table 1). Biofilm production was not significantly different amongst isolates from clinical infections compared with isolates obtained from colonized dogs $(\mathrm{p}=0.08)$ (Table 1$)$.

icaA was detected in 109/140 (77.9\%) of isolates (MRSP - 94/121 and MSSP - 15/19). No association between the presence of $i c a \mathrm{~A}$ and $\mathrm{OD}_{570}$ value $(\mathrm{P}=0.77)$, being a strong $(\mathrm{P}=0.08)$ or strong/moderate biofilm producer $(\mathrm{P}=0.15)$, and between MRSP and MSSP isolates $(\mathrm{P}=1.0)$ were detected.

icaD was detected in 106/140 (75.7\%) of isolates (MRSP - 93/121 and MSSP - 13/19). No association

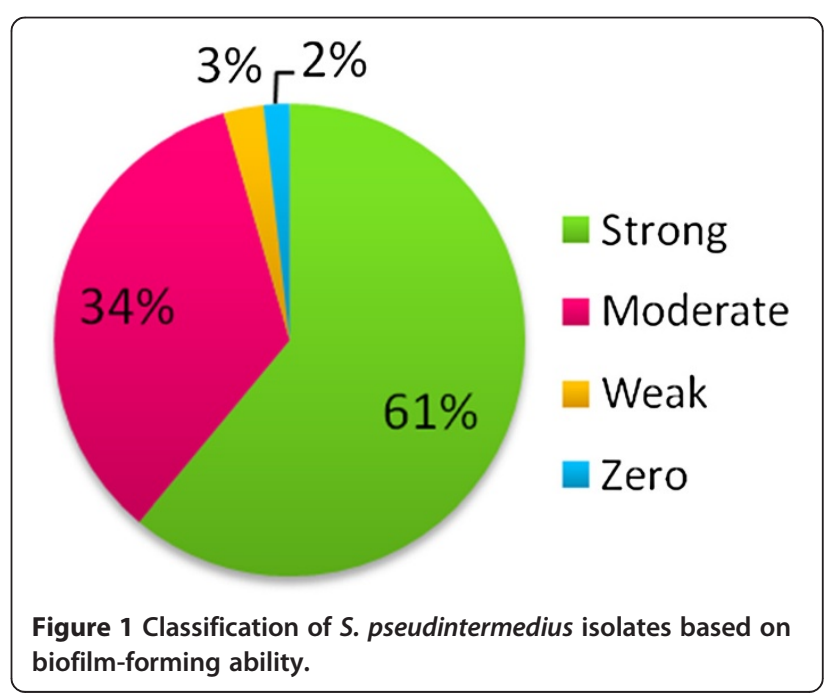


Table 1 Mean $\mathrm{OD}_{570}+/-$ standard deviation (SD) of various categories of Scxx pseudintermedius isolates evaluated

\begin{tabular}{|c|c|}
\hline Categorization (n) & Mean $\mathrm{OD}_{570}+/-\mathrm{SD}$ \\
\hline MRSP (121) & $0.75+/-0.57$ \\
\hline MSSP (19) & $0.74+/-0.41$ \\
\hline Infection (54) & $0.80+/-0.58$ \\
\hline Colonization (86) & $0.65+/-0.48$ \\
\hline ST 68 (55) & $0.79+/-0.64$ \\
\hline ST 71 (58) & $0.62+/-0.38$ \\
\hline
\end{tabular}

between the presence of $i c a \mathrm{D}$ and $\mathrm{OD}_{570}$ value $(\mathrm{P}=0.47)$, being a strong $(\mathrm{P}=0.97)$ or strong/moderate biofilm producer $(\mathrm{P}=0.26)$, and between MRSP and MSSP isolates $(\mathrm{P}=0.40)$ were detected.

Scanning electron microscopic evaluation of $S$. pseudintermedius biofilm production (Figures 2 and 3) on $316 \mathrm{~L}$ orthopaedic bone screws revealed the presence of aggregates of cocci in addition to large amounts of irregularly produced EPS.

\section{Discussion}

The majority of S. pseudintermedius isolates evaluated in this study were able to produce biofilm, with $96 \%$ being classified as either strong or moderate biofilm producers. Biofilm production by S. pseudintermedius may play an important role in the pathophysiology of disease and

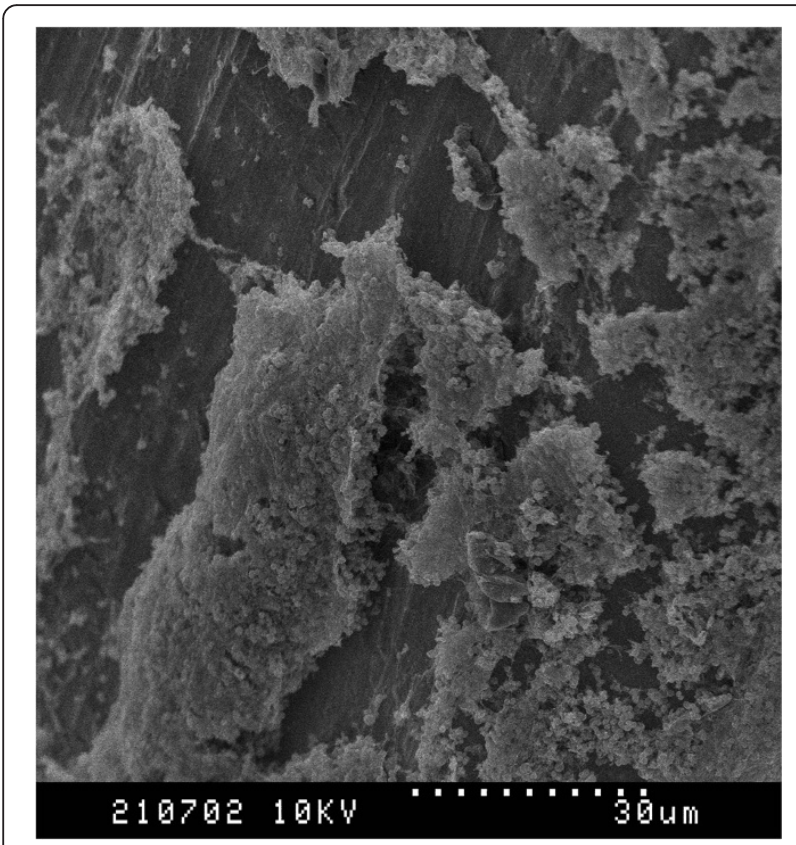

Figure 2 SEM Images of MRSP biofilm formation on 316L stainless steel orthopedic bone screws. Dotted line $=30 \mathrm{um}$ Large aggregates of cocci and irregularly produced extracellular polymeric substance are apparent.

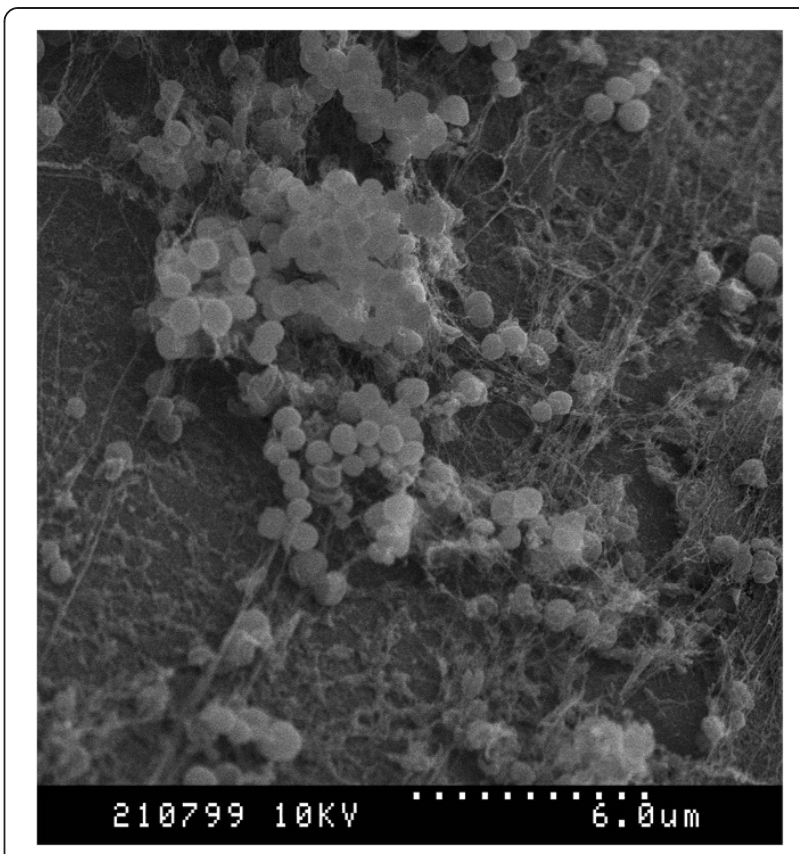

Figure 3 SEM Images of MRSP biofilm formation on 316L stainless steel orthopedic bone screws. Dotted line $=6 \mathrm{um}$. Large aggregates of cocci and irregularly produced extracellular polymeric substance are apparent.

potentially colonization, and could be a contributing factor in the rapid, worldwide emergence of MRSP [6,22]. Biofilm production has been correlated with clinical infection in other Staphylococcus spp., and further study into the role of biofilm formation in S. pseudintermedius is required [20,21].

Biofilm formation was not different between isolates of MRSP and MSSP, which may correspond to their equivalent virulence clinically [33]; however, the number of MSSP isolates that was studied was low. Regardless of methicillin resistance, biofilm formation may play a role in clinical infection with S. pseudintermedius, and further study into the biofilm forming ability of MSSP with a larger number of isolates is warranted.

S. pseudintermedius isolates from dogs with clinical infections did not have a significantly different biofilm forming ability compared with isolates from colonized dogs. This is not surprising given the similar distribution of MRSP clones amongst both infection and colonization isolates, and the fact that strains present on an individual (colonization) are presumably the source of infection. Biofilm formation may also be an important virulence factor allowing for colonization of $S$. pseudintermedius in dogs, facilitating survival in the upper respiratory tract and other body sites.

Investigation into the molecular epidemiology of isolates evaluated revealed the predominance of dru clusters 11a and 9a, corresponding to ST68 and ST71. These 
dru types are also the two most common clones in North America [34]. A recent European study investigated the biofilm forming ability of 23 MRSP isolates and reported significantly greater in vitro biofilm production by ST71 compared to other strains [22]. The same was not noted here; however, it must be noted that ST68 was not evaluated in that study. It is possible that both ST68 and ST71 are abundant biofilm producers. This could be a potential reason for why these two clones have emerged internationally as predominant MRSP clones amongst a highly diverse MSSP population [6,23].

The majority of $S$. pseudintermedius isolates contained $i c a \mathrm{~A}$ and icaD, which is consistent with a study in $S$. epidermidis which showed that isolates producing some level of biofilm using an MPA contained some genes of the ica operon [28]. Yet, there was no apparent association between the presence of either gene and biofilm production or methicillin resistance. Biofilm formation in staphylococci is complex and further study into the role of the ica operon and other potential genes in $S$. pseudintermedius is warranted.

The SEM images obtained after incubation of a strong biofilm forming isolate of $S$. pseudintermedius revealed biofilm formation on an orthopaedic implant. The biofilm was characterized with several aggregates of bacterial cells along with large amounts of amorphous EPS. This information provides visual evidence that S. pseudintermedius can form biofilm rapidly (within $24 \mathrm{hrs)}$ on stainless steel in an in vitro setting and is likely the same in vivo following placement of a surgical implant.

\section{Conclusion}

The majority of S. pseudintermedius isolates evaluated in this study were able to produce biofilm and this may be an important virulence factor in the rapid emergence of this bacterium in veterinary hospitals worldwide. There appears to be no association between biofilm formation and methicillin resistance, infection vs colonization source and clonal complex. As with other staphylococci, ica produced PIA may play a role in S. pseudintermedius biofilm formation, but the exact mechanism of biofilm formation in S. pseudintermedius requires further study.

\section{Competing interests}

The authors declare that they have no competing interests.

\section{Authors' contributions}

AS and JSW were responsible for study design and manuscript preparation. MW and JR performed microbiological testing. All authors read and approved the final manuscript.

\section{Author details}

${ }^{1}$ Department of Clinical Studies, Ontario Veterinary College, University of Guelph, Guelph, ON N1G 2W1, Canada. ²Department of Pathobiology, Ontario Veterinary College, University of Guelph, Guelph, ON N1G 2W1, Canada.

Received: 21 December 2012 Accepted: 1 May 2013

Published: 3 May 2013

\section{References}

1. Vasseur PB, Levy J, Dowd E, Eliot J: Surgical wound infection rates in dogs and cats. Data from a teaching hospital. Vet Surg 1988, 17:60-64.

2. Nicholson M, Beal M, Shofer F, Brown DC: Epidemiologic evaluation of postoperative wound infection in clean-contaminated wounds: a retrospective study of 239 dogs and cats. Vet Surg 2002, 31:577-581.

3. Eugster S, Schawalder P, Gaschen F, Boerlin P: A prospective study of postoperative surgical site infections in dogs and cats. Vet Surg 2004, 33:542-550.

4. Brown DC, Conzemius MG, Shofer F, Swann H: Epidemiologic evaluation of postoperative wound infections in dogs and cats. J Am Vet Med AssoC 1997, 210:1302-1306.

5. Weese JS: A review of multidrug resistant surgical site infections. Vet Comp Orthop Traumatol 2008, 21:1-7.

6. Perreten V, Kadlec K, Schwarz S, et al: Clonal spread of methicillin-resistant Staphylococcus pseudintermedius in Europe and North America: an international multicenter study. J Antimicrob Chemother 2010, 65:1145-1154.

7. Nienhoff U, Kadlec K, Chaberny IF, et al: Methicillin-resistant Staphylococcus pseudintermedius among dogs admitted to a small animal hospital. Vet Microbiol 2011, 150:191-197.

8. Ruscher C, Lubke-Becker A, Semmler T, et al: Widespread rapid emergence of a distinct methicillin- and multidrug-resistant Staphylococcus pseudintermedius isolated from dogs. Vet Microbiol 2010, 144:340-346.

9. Singh A, Turk R, Weese JS: Post-discharge procedure specific surgical site infection surveillance in small animals. Barcelona, Spain: Proceedings of the European College of Veterinary Surgery Symposium; 2012.

10. Thompson AM, Bergh MS, Wang C, Wells K: Tibial plateau leveling osteotomy implant removal: a retrospective analysis of 129 cases. Vet Comp Orthop Traumatol 2011, 24:450-456.

11. Gallagher AD, Mertens WD: Implant removal rate from infection after tibial plateau leveling osteotomy in dogs. Vet Surg 2012, 41:705-711.

12. Weese JS: A review of post-operative infections in veterinary orthopedic surgery. Vet Comp Orthop Traumatol 2008, 21:99-105.

13. Vos D, Hanson B, Verhofstad M: Implant removal of osteosynthesis: the Dutch practice. Results of a survey. J Trauma Manag Outcomes 2012, 6:6.

14. Zimmerli W, Moser C: Pathogenesis and treatment concepts of orthopaedic biofilm infections. FEMS Immunol Med Microbiol 2012, 65:158-168.

15. Costerton JW, Montanaro L, Arciola CR: Biofilm in implant infections: its production and regulation. Int J Art Org 2005, 28:1062-1068.

16. Flemming HC, Wingender J: The biofilm matrix. Nat Rev Microbio/ 2010, 8:623-633.

17. Costerton JW: Biofilm theory can guide the treatment of device-related orthopedic infections. Clin Orthop Rel Res 2005, 437:7-11.

18. Montanaro L, Speziale P, Campoccia D, et al: Scenery of Staphylococcus implant infections in orthopedics. Future Microbiol 2002, 6:1329-1349.

19. Donlan R: Biofilms: microbial life on surfaces. Emerg Inf Dis 2011, 8:881-890.

20. Jain A, Agarwal A: Biofilm production, a marker of pathogenic potential of colonizaing and commensal staphylococci. J Microbiol Meth 2009, 76:88-92.

21. Nayak N, Satpathy G, Nag HL, et al: Slime production is essential for the adherence of Staphylococcus epidermidis in implant-related infections. $J$ Hosp Inf 2011, 77:152-156.

22. Osland AM, Vestby LK, Fanuelsen $H$, et al: Clonal diversity and biofilmforming ability of methicillin-resistant Staphylococcus pseudintermedius. J Antimicrob Chemother 2012, 67:841-848.

23. Dicicco M, Neethirajan S, Singh A, Weese JS: Efficacy of clarithromycin on biofilm formation of methicillin-resistant Staphylococcus pseudintermedius. BMC Vet Res 2012, 8:225.

24. Rohde H, Frankenberger S, Zahringer U, Mack D: Structure, function and contribution of polysaccharide intercellular adhesion (PIA) to Staphylococcus epidermidis biofilm formation and pathogenesis of biomaterial-associated infections. Eur J Cell Bio 2010, 89:103-111.

25. Gerke C, Kraft A, Sussmuth R, et al: Characterization of the $\mathrm{N}$ acetylglucosaminyltransferase activity involved in the biosynthesis of the Staphylococcus epidermidis polysaccharide intercellular adhesin. J Biol Chem 1998, 273:18586-18593.

26. Fitzpatrick F, Humphreys $\mathrm{H}, \mathrm{O}^{\prime} \mathrm{Gara} J \mathrm{~J}$ : Evidence for icaADBC-independent biofilm development mechanism in methicillin-resistant Staphylococcus aureus clinical isolates. J Clin Microbiol 2005, 43:1973-1976.

27. Stepanovich S, Vukovic D, Hola V, et al: Quantification of biofilm in microtiter plates: overview of testing conditions and practical 
recommendations for assessment of biofilm production by staphylococci. APMIS 2007, 115:891-899.

28. Arciola CR, Baldassarri L, Montanaro L: Presence of icaA and icaD and slime production in a collection of staphylococcal strains from catheterassociated infections. J Clin Microbiol 2001, 39:2151-2156.

29. Arciola CR, Collamati S, Donati E, Montanaro L: A rapid PCR-method for the detection of slime-producing strains of Staphylococcus epidermidis and Staphylococcus aureus in periprosthesis infections. Diagn Mol Pathol 2001, 10:130-137.

30. Goering RV, Morrison D, Al-Doori Z, Edwards GF, Gemmell CG: Usefulness of mec-associated direct repeat unit (dru) typing in the epidemiological analysis of highly clonal methicillin-resistant Staphylococcus aureus in Scotland. Clin Microb Infect 2008, 14:964-969.

31. Shore AC, Rossney AS, Kinnevey PM, et al: Enhanced discrimination of highly clonal ST22-methicillin-resistant Staphylococcus aureus IV isolates achieved by combining spa, dru, and pulsed-field gel electrophoresis typing data. J Clin Microbiol 2010, 48:1839-1852.

32. Weese JS, Rousseau J, Kadlec K, et al: Direct repeating unit (dru) typing of methicillin-resistant Staphylococcus pseudintermedius from North America and Europe. Proceedings. San Francisco, CA: International Society for Companion Animal Infectious Disease Annual Conference; 2012.

33. Weese JS, Faires MC, Frank LA, Reynolds LM: Factors associated with methicillin-resistant versus methicillin-susceptible Staphylococcus pseudintermedius infection in dogs. J Am Vet Med Assoc 2012, 240:1450-1455.

34. Weese JS, Sweeman K, Edson H, Rousseau J: Evaluation of minocycline susceptibility of methicillin-resistant Staphylococcus pseudintermedius. Vet Micro 2012. Epub ahead of print.

doi:10.1186/1746-6148-9-93

Cite this article as: Singh et al: Characterization of the biofilm forming ability of Staphylococcus pseudintermedius from dogs. BMC Veterinary Research 2013 9:93.

\section{Submit your next manuscript to BioMed Central and take full advantage of:}

- Convenient online submission

- Thorough peer review

- No space constraints or color figure charges

- Immediate publication on acceptance

- Inclusion in PubMed, CAS, Scopus and Google Scholar

- Research which is freely available for redistribution 